\title{
Novel phases and transitions in Color Flavor Locked matter
}

\author{
David B. Kaplan[ and Sanjay Reddy" \\ Institute for Nuclear Theory \\ University of Washington, Box 351550, Seattle, WA 98195-1550, USA
}

\begin{abstract}
We analyze the phases of dense quark matter at nonzero quark masses. We map out the phase diagrams for charged quark matter as well as charge neutral matter containing leptons. We find that in Color Flavor Locked (CFL) quark matter, the symmetric phase with equal numbers of $u, d$ and $s$ quarks is unlikely to occur in nature. Various less symmetric phases with additional meson condensates may play a role in neutron star cores, however, in agreement with the analysis of Bedaque and Schäfer. We discuss the implications of these novel color superconducting quark matter phases and their transitions for neutron stars.
\end{abstract}

\footnotetext{
${ }^{1}$ dbkaplan@phys . washington. edu

${ }^{2}$ reddy@phys . washington. edu
} 


\section{The meson condensed phases}

There has recently been renewed interest in the properties of dense quark matter [1, 2]. Three flavor QCD with massless quarks at high baryon density is thought to have a particularly symmetric ground state, the Color Flavor Locked (CFL) phase [3]. In this phase the $S U(3)_{\text {color }} \times S U(3)_{L} \times$ $S U(3)_{R} \times U(1)_{B}$ symmetry of QCD is broken down to the global diagonal $S U(3)$ symmetry due to BCS-like pairing between quarks near the Fermi surface ${ }^{6}$. As a result, the gluons become massive via the Higgs mechanism. There is the possibility that color superconductivity is relevant to the structure of neutron stars. However, in realistic situations, quark masses are nonzero and there may be nonzero chemical potentials for charge or lepton number, and the exact flavor symmetry of the QCD Lagrangian is reduced to $U(1)_{e m} \times U(1)_{Y} \times U(1)_{B}$, in which case one might expect the CFL condensate to preserve the $U(1)_{e m} \times U(1)_{Y}$ subgroup of $S U(3)$ not explicitly brokenf. One can best understand how the symmetric CFL phase distorts under these applied stresses by studying the effective theory for the light excitations [5, 6, 7, 8, 9, 10, 11], the subject of this Letter. In particular, we explore the phase diagram for realistic quark masses in a density regime that may occur in neutron star interiors, exploiting recent observations by Schäfer and Bedaque [12. We find that the symmetric CFL phase is unstable with respect to meson condensation, except above baryon densities approximately $10^{21}$ times that which might be expected in a neutron star core. Meson condensation results in a number of less symmetric color superconducting phases that may be relevant, with implications for the thermodynamic and transport properties of neutron star cores.

The low lying spectrum of the symmetric CFL phase with massless quarks consists of a nonet of massless Goldstone bosons transforming under the unbroken $S U(3)$ as an octet plus a singlet. A tenth light meson is also expected, a pseudo-Goldstone boson arising from spontaneously broken axial

\footnotetext{
${ }^{3} S U(3)_{\text {color }}$ is the gauged symmetry of QCD; $S U(3)_{L} \times S U(3)_{R}$ is the global symmetry which acts on the three flavors of left- and right-handed quarks; $U(1)_{B}$ is the baryon number symmetry.

${ }^{4}$ Throughout this paper we will refer to both the $S U(3)$ symmetric phase in the absence of quark masses and chemical potentials, and to the $U(1)_{e m} \times U(1)_{Y}$ symmetric phase in the presence of masses and chemical potentials, as the "symmetric CFL phase". The identifying characteristic of the symmetric CFL ground state is that it contains equal numbers of $u, d$ and $s$ [ $[$ ].
} 
$U(1)_{A}$ - an approximate symmetry broken by instanton effects, which are expected to be weak at high density. The massless mesons allow the system to respond continuously to applied stresses, such as the imposition of nonzero chemical potentials for $S U(3)$ or $U(1)_{B}$ currents, since there is no gap in these channels for massless quarks.

Turning on nonzero quark masses induces a gap in the spectrum. This would seem to make the symmetric CFL phase rigid against distortion due to applied chemical potentials. However, as recently pointed out by Schäfer and Bedaque [12], while quark masses produce gaps in the spectrum, they also induce new stresses on the system, acting in a manner analogous to an applied chemical potential and favoring meson condensation. As discussed by those authors, nonzero quark masses can be expected to lead to meson condensation and a complicated phase diagram.

We can parameterize the effective theory describing excitations about the $S U(3)$ symmetric CFL ground state in terms of the two fields $B=$ $e^{i \beta / f_{B}}$ and $\Sigma=e^{2 i\left(\pi / f_{\pi}+\eta^{\prime} / f_{A}\right)}$, representing the Goldstone bosons of broken baryon number $\beta$, and of broken chiral symmetry, the pseudoscalar octet $\pi$, and the pseudo-Goldstone boson $\eta^{\prime}$, arising from broken approximate $U(1)_{A}$ symmetry. Color degrees of freedom have been integrated out, and so under the original global $S U(3)_{L} \times S U(3)_{R} \times U(1)_{B} \times U(1)_{A}$ symmetry, these fields transform as the representations $B=(1,1)_{1,0}$ and $\Sigma=(3, \overline{3})_{0,-4}$.

The quark mass matrix $M=\operatorname{diag}\left(m_{u}, m_{d}, m_{s}\right)$ acts like a spurion, transforming as the representation $(3, \overline{3})_{0,2}$. Due to the $U(1)_{A}$ charges, operators in the effective theory must involve even powers of $M$. Although there are two independent operators one can construct with two powers of $M$, the leading operator at weak coupling involves only the combination $\tilde{M} \equiv|M| M^{-1}=\operatorname{diag}\left(m_{d} m_{s}, m_{s} m_{u}, m_{u} m_{d}\right)$, which transforms as a $(\overline{3}, 3)_{0,4}$ and can couple to $\Sigma$ as $\operatorname{Tr} \tilde{M} \Sigma$. It is this operator that gives the leading contribution to the meson masses. However, one can also consider operators involving one power of $M$ and one power of $M^{\dagger}$. For example, the traceless part of $M^{\dagger} M$, transforms as $(8,1)_{0,0}$, the same as a left-handed flavor current. It has recently been pointed out by Bedaque and Schäfer that the combination $M^{\dagger} M /(2 \mu)$ (where $\mu$ is the quark number chemical potential) enters the effective theory exactly as would a chemical potential for flavor symmetries [12] ․ As they discuss, this term destabilizes the ground state for sufficiently large strange quark mass and leads to kaon condensation.

\footnotetext{
${ }^{5}$ A similar observation was made in Ref. 10]
} 
The leading terms in the effective Lagrangian describing spatially constant nonet Goldstone boson fields $\pi$ and $\eta^{\prime}$ in the presence of a chemical potential $\mu_{Q}$ for electric charge is given by

$$
\begin{aligned}
\mathcal{L}= & f_{\pi}^{2}\left[\frac{1}{4} \operatorname{Tr} D_{0} \Sigma D_{0} \Sigma^{\dagger}+\frac{1}{2} \frac{f_{A}^{2}}{f_{\pi}^{2}}\left(D_{0} \eta^{\prime}\right)^{2}+\frac{a}{2} \operatorname{Tr} \tilde{M}\left(\Sigma+\Sigma^{\dagger}\right)+\frac{b}{2} \operatorname{Tr} Q \Sigma Q \Sigma^{\dagger}\right] \\
& D_{0} \Sigma=\partial_{0} \Sigma-i\left[\left(\mu_{Q} Q+X_{L}\right) \Sigma-\Sigma\left(\mu_{Q} Q+X_{R}\right)\right] .
\end{aligned}
$$

The decay constants $f_{\pi}$ and $f_{A}$ have been computed previously [7]. $Q$ is the electric charge matrix $\operatorname{diag}(2 / 3,-1 / 3,-1 / 3)$ while $X_{L, R}$ are the BedaqueSchäfer terms: $X_{L}=-\frac{M M^{\dagger}}{2 \mu}, \quad X_{R}=-\frac{M^{\dagger} M}{2 \mu}$, and $\tilde{M}=|M| M^{-1}$.

The leading contributions to meson masses are the $a$ and $b$ operators. The coefficient $a$ has been computed and is given by $a=3 \frac{\Delta^{2}}{\pi^{2} f_{\pi}^{2}}$ [] (for a discussion of possible log corrections see [9, 10, 11]). The $b$ term accounts for electromagnetic corrections to the charged meson masses. The coefficient $b$ is proportional to $\tilde{\alpha}$ (the fine structure constant for the massless photon in the $S U(3)$ symmetric CFL phase) and its value is scheme dependent $\mathrm{F}$. From naive dimensional analysis, one can estimate the contribution to $b$ from photon loops in the effective theory, leading to $b \sim \frac{\tilde{\alpha}}{4 \pi} \Delta^{2}$; this formula reflects the quadratic divergence of a photon loop in the effective theory, which has a momentum cutoff equal to the gap $\Delta$. This estimate is consistent with that of [14, given that that reference did not attempt to estimate the factors of $4 \pi$. If the correct value of $b$ differs from the crude estimate given above, our results will be changed quantitatively but not qualitatively.

The meson masses in terms of the parameters $a, b$ are

$$
\begin{aligned}
m_{\pi^{-}}^{2} & =a\left(m_{u}+m_{d}\right) m_{s}+b \\
m_{K^{-}}^{2} & =a\left(m_{u}+m_{s}\right) m_{d}+b \\
m_{K^{0}}^{2} & =a\left(m_{d}+m_{s}\right) m_{u} .
\end{aligned}
$$

The free energy density corresponding to the Lagrangian in eq. (11) in the mean field approximation, relative to the free energy density of the symmetric CFL phase (corresponding to $\Sigma=\mathbf{1}$ ) is:

$$
\Omega=\frac{f_{\pi}^{2}}{4}\left[\operatorname{Tr}[\tilde{\mu}, \Sigma]\left[\tilde{\mu}, \Sigma^{\dagger}\right]-2 a \operatorname{Tr} \tilde{M}\left(\Sigma+\Sigma^{\dagger}-2\right)-b \operatorname{Tr}[Q, \Sigma]\left[Q, \Sigma^{\dagger}\right]\right],
$$

\footnotetext{
${ }^{6}$ If one uses a mass independent subtraction scheme in Landau gauge, the one-loop electromagnetic corrections to the meson masses vanish, and the $b$ term alone accounts for the entire electromagnetic splittings. We assume this scheme, and do not include explicit photon fields.
} 
where $\Sigma$ is a constant $\mathrm{U}(3)$ matrix characterizing the ground state. Working in the standard basis where the quark mass matrix is real, the matrix $\tilde{\mu}$ is given by

$$
\tilde{\mu}=\mu_{Q}+X, \quad X=X_{L}=X_{R}=-\frac{M^{2}}{2 \mu} .
$$

The effective chemical potentials vanish for the $\pi^{0}, \eta$ and $\eta^{\prime}$, while for the $\pi^{+}, \mathrm{K}^{+}$and $\mathrm{K}^{0}$ mesons they are

$$
\tilde{\mu}_{\pi^{+}}=\mu_{Q}+\frac{m_{d}^{2}-m_{u}^{2}}{2 \mu}, \quad \tilde{\mu}_{K^{+}}=\mu_{Q}+\frac{m_{s}^{2}-m_{u}^{2}}{2 \mu}, \quad \tilde{\mu}_{K^{0}}=\frac{m_{s}^{2}-m_{d}^{2}}{2 \mu} .
$$

The stationary points of the free energy $\Omega$ with respect to variations of the meson fields are found as solutions to the matrix equation

$$
\left[\tilde{\mu} \Sigma^{\dagger} \tilde{\mu} \Sigma-a M \Sigma-b Q \Sigma^{\dagger} Q \Sigma\right]-\text { h.c. }=0 .
$$

To find solutions to this equation, we begin by considering $\Sigma$ matrices corresponding to a condensate of a single meson $\langle\phi\rangle \equiv \sqrt{2} f_{\pi} \theta$, where $\phi$ could be a charged pion, a charged kaon, or a neutral kaon field. Solving eq. (6) yields stationary points with $\theta \neq 0$ which for certain ranges of parameters have lower free energy than the symmetric CFL phase $(\theta=0)$. We find the solutions

$$
\begin{array}{ll}
\Omega_{\pi^{ \pm}}=-\frac{f_{\pi}^{2}}{2}\left(\tilde{\mu}_{\pi^{ \pm}}^{2}-b\right)\left(1-\cos \theta_{\pi^{ \pm}}\right)^{2}, & \cos \theta_{\pi^{ \pm}}= \begin{cases}1 & M_{\pi^{ \pm}}^{2} \geq \tilde{\mu}_{\pi^{ \pm}}^{2} \\
\frac{M_{\pi \pm}^{2}-b}{\tilde{\mu}_{\pi^{ \pm}}^{2}-b} & M_{\pi^{ \pm}}^{2} \leq \tilde{\mu}_{\pi^{ \pm}}^{2}\end{cases} \\
\Omega_{K^{ \pm}}=-\frac{f_{\pi}^{2}}{2}\left(\tilde{\mu}_{K^{ \pm}}^{2}-b\right)\left(1-\cos \theta_{K^{ \pm}}\right)^{2}, & \cos \theta_{K^{ \pm}}= \begin{cases}1 & M_{K^{ \pm}}^{2} \geq \tilde{\mu}_{K^{ \pm}}^{2} \\
\frac{M_{K^{ \pm}}^{2}-b}{\tilde{\mu}_{K^{ \pm}}^{2}-b} & M_{K^{ \pm}}^{2} \leq \tilde{\mu}_{K^{ \pm}}^{2}\end{cases} \\
\Omega_{K^{0}}=-\frac{f_{\pi}^{2}}{2} \tilde{\mu}_{K^{0}}^{2}\left(1-\cos \theta_{K^{0}}\right)^{2}, & \cos \theta_{K^{0}}= \begin{cases}1 & M_{K^{0}}^{2} \geq \tilde{\mu}_{K^{0}}^{2} \\
\frac{M_{K^{0}}^{2}}{\tilde{\mu}_{K^{0}}^{2}} & M_{K^{0}}^{2} \leq \tilde{\mu}_{K^{0}}^{2}\end{cases}
\end{array}
$$

Evidently the nontrivial solutions with $\theta \neq 0$ represent phases with lower free energy than the symmetric CFL phase. The charged pion and kaon phases 
can have either electric charge, depending on the sign of appropriate effective chemical potential $\tilde{\mu}$. We will refer to these 3-flavor, color superconducting, meson condensed phases as $\mathrm{CFL} \pi^{ \pm}, \mathrm{CFLK}^{ \pm}$and $\mathrm{CFLK}^{0}$ respectively, or simply as $\pi^{ \pm}, \mathrm{K}^{ \pm}$and $\mathrm{K}^{0}$, distinguishing them from the more symmetric CFL phase.

\section{The phase diagram}

In Fig. 1 we plot the phase diagram for color superconducting quark matter, as a function of $m_{s}^{2} /(2 \mu)$, where $\mu$ is the quark number chemical potential, versus the chemical potential for positive electric charge, $\mu_{Q}$. In order to construct this diagram we used the quark mass ratios derived from chiral perturbation theory [15], $m_{u} / m_{d}=1 / 2, m_{d} / m_{s}=1 / 20$, with the additional assumption $m_{s}=150 \mathrm{MeV}$. For the parameter $b$ in the chiral Lagrangian, we used the rough estimate $b=\frac{\alpha}{4 \pi} \Delta^{2}$. Finally, for the relation between the color superconducting gap $\Delta$ and $\mu$, we used the perturbative expression from Ref.([16]) down to $\mu=10 \mathrm{GeV}$. At lower densities, we choose a smooth interpolant that mimics phenomenological models and predicts $\Delta \sim 100 \mathrm{MeV}$ for $\mu \sim 500 \mathrm{MeV}$ [1]. The superconducting gap obtained in this way is shown in Fig. 2. We note that, even in the perturbative regime, the numerical value of the gap is still not well understood due to ambiguities associated with scale dependence of the gap equation [17]. However, we find that although the precise location of the phase boundaries in Fig. 1 depend on the numerical value of the gap, the qualitative features of the phase diagram, which will be discussed below, are fairly independent of $\Delta(\mu)$.

Several features of the phase diagram Fig. 1 are striking. The first is that the symmetric CFL phase is disfavored until quark densities greater than $\sim 10^{21}$ times what one might find in neutron star cores. Instead, at realistic values for $\mu$, the $\mathrm{K}^{0}$ phase is favored for small $\mu_{Q}$, while the $\mathrm{K}^{+}$and $\pi^{-}$phases are favored for $\mu_{Q}$ above or below critical values. The $\overline{\mathrm{K}}^{0}$ and $\mathrm{K}^{-}$condensed phases are disfavored as they introduce strange quarks in a system trying to reduce their number 7 , while for positive $\mu_{Q} \mathrm{~K}^{+}$is favored over $\pi^{+}$both because the $\mathrm{K}^{+}$is lighter, and because a $\mathrm{K}^{+}$condensate reduces the strange quark number while a pion condensate does not.

\footnotetext{
${ }^{7}$ This is in contrast to kaon condensation in hadronic matter 18, 19], which suffers from a paucity of strange quarks, while the symmetric CFL phase has a surplus.
} 
As one approaches from either side the boundaries between the $\mathrm{K}^{0}$ phase and the $\mathrm{K}^{+}$or $\pi^{-}$phases, one finds that the meson condensate angles $\theta$ given in eq. (7) do not vanish. This indicates that the phase transitions are first order. One can verify this by solving the stationarity equation eq. (6) with a two condensate ansatz for the $\Sigma$ matrix. As an example we analyze the $\mathrm{K}^{+} / \mathrm{K}^{0}$ transition. With the ansatz

$$
\Sigma=\exp \left[i \theta\left(\begin{array}{ccc}
0 & 0 & \sin \phi \\
0 & 0 & \cos \phi \\
\sin \phi & \cos \phi & 0
\end{array}\right)\right]
$$

one finds a saddle point solution to eq. (6) interpolating between the $\mathrm{K}^{0}$ and $\mathrm{K}^{+}$phases, given by

$$
\begin{aligned}
\cos \theta & =\frac{M_{K^{0}}^{2}}{\tilde{\mu}_{K^{0}}^{2}}+\frac{\tilde{\mu}_{K^{+}}\left(\tilde{\mu}_{K^{+}}-\tilde{\mu}_{K^{0}}\right)}{b \tilde{\mu}_{K^{0}}}\left[\left(\frac{M_{K^{+}}^{2}-\tilde{\mu}_{K^{+}}^{2}}{\tilde{\mu}_{K^{+}}}\right)-\left(\frac{M_{K^{0}}^{2}-\tilde{\mu}_{K^{0}}^{2}}{\tilde{\mu}_{K^{0}}}\right)\right], \\
\sin ^{2} \phi & =\frac{\left(\frac{M_{K^{0}}^{2}}{\tilde{\mu}_{K^{0}}^{2}}-\cos \theta\right)}{\left(1-\frac{\tilde{\mu}_{K^{+}}}{\tilde{\mu}_{K^{0}}}\right)(1-\cos \theta)} .
\end{aligned}
$$

This saddle point solution exists only for ranges of parameters for which the angles in eq. (9) are real. The free energy density of this solution relative to that of the $\mathrm{K}^{0}$ phase is

$$
\delta \Omega \equiv\left(\Omega_{K^{0} / K^{+}}-\Omega_{K^{0}}\right)=\frac{f_{\pi}^{2}}{4} \frac{\left(m_{K^{0}}^{2}-\mu_{k^{0}}^{2} \cos \theta\right)^{2}}{\mu_{K^{0}}^{2}\left(\mu_{K^{0}}-\mu_{K^{+}}\right)^{2}} b .
$$

It is the quantity $\delta \Omega$ which will control the tunneling rate between the $\mathrm{K}^{+}$ and $\mathrm{K}^{0}$ phases during a first order phase transition.

\section{$3 \quad$ Neutron stars}

If quark matter were to exist in neutron stars, the typical quark chemical potential is $\mu \sim 400 \mathrm{MeV}$. As discussed earlier, perturbative calculations are inapplicable here and model calculations based on phenomenological fourfermion interactions suggest that three flavor massless quark matter may be characterized by a gap as large as $\Delta \sim 100 \mathrm{MeV}$. As will become clear from the following discussion, this is larger than the typical energy associated with 
external stresses that quark matter is subject to in the neutron star interior. Therefore we can calculate the response of quark matter to these stresses within the frame work of the effective theory defined by eq. (1).

Neutron stars are born in the aftermath of a core collapse supernova explosion. The inner core of the newly born neutron star is characterized by a temperature $\mathrm{T} \sim 30 \mathrm{MeV}$ and a lepton fraction (lepton number/baryon number) $\mathrm{Y}_{\mathrm{L}} \sim 0.3$ implying $\mu_{e} \equiv \mu_{\nu_{e}}-\mu_{Q} \sim \mu_{\nu_{e}} \sim 200 \mathrm{MeV}$. The high temperature and finite lepton chemical potentials are a consequence of neutrino trapping during gravitational collapse [20]. Subsequently, the interior cools and looses lepton number via neutrino diffusion. This occurs on a time scale of few tens of seconds. At later times, $\mathrm{T} \ll 1 \mathrm{MeV}, \mu_{\nu_{e}}=0$ and the electron chemical potential is determined by the condition of electric charge neutrality. In noninteracting quark matter, $\mu_{e} \sim m_{s}^{2} / 2 \mu \sim 25 \mathrm{MeV}$. However, in the superconducting phase with $\Delta \gtrsim m_{s}^{2} / 4 \mu$ our phase diagram Fig. 1 indicates that the ground state is the $\mathrm{K}^{0}$ phase. The $\mathrm{K}^{0}$ phase is electrically neutral without electrons. This result has far reaching consequences because, being devoid of electrons, the quark phase has no low lying charged excitations up to the mass of the lightest charged Goldstone boson. In the symmetric CFL phase, neutrality is guaranteed by the enforced equality of the $\mathrm{u}, \mathrm{d}$, and $\mathrm{s}$ quarks [4]. For the $\mathrm{K}^{0}$ phase, charge neutrality is a fluke and is due to the fact that the $\mathrm{K}^{+}$is heavier than the $\mathrm{K}^{0}$.

The core of a neutron star during the early stages of its evolution will be charge neutral, and will have nonzero lepton number. Assuming local electric charge neutrality, the possible ground states among the phases we have discussed are $\mathrm{K}^{0}$ condensation with all the leptons being neutrinos; $\mathrm{K}^{+}$condensation with charge neutralizing electrons; or a mixed $\mathrm{K}^{+} / \mathrm{K}^{0}$ condensate with electrons, corresponding to $0<\phi<\pi / 2$ in eq. (8). Our analysis at fixed $\mu_{Q}$ found that the latter phase was not a minimum of the free energy, but a saddle point. Remarkably enough, when leptons are included and local charge neutrality is imposed, the saddle point solution of eq. (11) becomes the true minimum of the free energy over the range that it exists. Further, we find that it smoothly connects the $\mathrm{K}^{0}$ phase with $\mathrm{K}^{+}$phase resulting in second order transition transitions. Note that eq. (6) remains valid and characterizes the stationary points of the free energy even when charge neutrality is imposed at fixed lepton chemical potential.

A phase diagram depicting each of the three phases discussed above are shown in Fig. 3. Electric charge neutrality is enforced in the phase containing the $\mathrm{K}^{+}$mesons by requiring that the electron number density 
$n_{e}=-\partial \Omega_{\text {meson }} / \partial \mu_{Q}$. This uniquely determines the electron chemical potential and hence their contribution to the total free energy. In Fig. 3 we restrict the quark chemical potential to the range of relevance for neutron stars: 400$1000 \mathrm{MeV}$; and make the simplifying assumption that the gap $\Delta=100 \mathrm{MeV}$ over this range.

From Fig. 3 we can infer that the ground state will contain electrons for $\mu_{\nu_{e}} \gtrsim 6 \mathrm{MeV}$. The superconducting phase admits electrons with ease since the $\mathrm{K}^{+}$mesons are not much heavier than the neutral $\mathrm{K}^{0}$ mesons. We find that the electric charge chemical potential $\mu_{Q}$ is only a few $\mathrm{MeV}$ even when $\mu_{\nu_{e}} \sim 200 \mathrm{MeV}$. Therefore the induced stress on the quark phase, characterized by $\tilde{\mu}_{\mathrm{K}^{+}}=\mu_{Q}+X$, is always small compared to $\Delta$. Consequently, unless excluded by high temperatures, quark matter in neutron star cores will be color superconducting even when leptons are trapped. As lepton number is lost via neutrino diffusion, we can expect a second order transition from the $\mathrm{K}^{+}$to the two condensate phase. Upon further deleptonization, when $\mu_{\nu_{e}} \sim 5-10 \mathrm{MeV}$ another second order transition from the $\mathrm{K}^{0} / \mathrm{K}^{+}$phase (containing electrons) to the $\mathrm{K}^{0}$ phase (without electrons) is to be expected.

Although it is premature to speculate precisely how this will influence various observable aspects of the early evolution of neutron stars we comment on a few important consequences of our findings. Firstly, since the only hadronic excitations in the CFL $\phi$ phases with energies below the gap are the pseudo Goldstone bosons with masses $\sim 10-20 \mathrm{MeV}$ the leptons are likely to dominate the transport processes and the matter specific heat. During deleptonization we can expect dramatic changes to these fore mentioned properties. This is likely to affect various aspects of the early evolution of the neutron star including neutrino transport.

If magnetic fields play a role during this early phase, we can expect field topology to change greatly during the early deleptonization phase since the initial $\mathrm{K}^{+}$and $\mathrm{K}^{0} / \mathrm{K}^{+}$phases are electrical superconductors they will expel the magnetic field in the core due to Meissner effect; while at later times when the system is characterized by a neutral $\mathrm{K}^{0}$ phase and the $\mathrm{U}(1)_{\mathrm{em}}$ is restored, the Meissner affect disappears and the core now admits the magnetic field.

Finally, since the neutrino free ground state contains no electrons, we note that the total lepton number emitted in neutrinos will be $20 \%-30 \%$ larger than in conventional scenarios. This is particularly, exciting because current

8 This depends on the value of $\mathrm{b}$, for example, for $b=e^{2} \Delta^{2}\left(16 \pi^{2}\right.$ times larger than the value we use) electrons appear for $\mu_{\nu_{e}} \gtrsim 20 \mathrm{MeV}$ 
neutrino detectors such as SNO, which can distinguish between different flavors, in conjunction with larger detectors, such as Super Kamiokande which primarily detect the anti-electron neutrinos, is in principle sensitive to the lepton number emitted from a galactic supernova.

It must be stressed that there are significant uncertainties in our analysis. Most importantly, the density regime one expects in neutron star cores is not high enough for the application of perturbative QCD. At these densities, the question of whether a quark phase exists at all, whether color superconductivity occurs should such a quark phase exist, and what the size of the resulting gap and chiral Lagrangian parameters would be, are all presently unanswerable. In our analysis we have extrapolated from the perturbative regime and respected the symmetries of QCD, with the exception that we have assumed $U(1)_{A}$ violating instanton effects to be small. If the latter assumptions proves to be false at the relatively low densities found in neutron stars, a $\operatorname{Tr} M \Sigma$ will be induced in the chiral Lagrangian [3, 10], altering our analysis. We also note that we have ignored finite temperature effects in our analysis of the phase diagram. Thus we require that the matter temperature be small compared to the superconducting critical temperature $\mathrm{T}_{\mathrm{c}} \sim \Delta$. In addition, we have neglected finite temperature corrections to the effective theory and the role of thermally excited mesons, which we expect to be small in the protoneutron star context. However, the contribution of thermal mesons to the free energy may not be negligible. Nevertheless, we feel confident that should color superconductivity exist in neutron stars, the phase diagram we have computed will be qualitatively correct and warrants further investigation.

\section{Acknowledgements}

We thank S. Beane, G. F. Bertsch, K. Rajagopal, M. Savage, and T. Schäfer for useful comments. This work was supported by DOE grant DEFG03-00-ER-41132. 


\section{References}

[1] M. Alford, K. Rajagopal and F. Wilczek, Phys. Lett. B422, 247 (1998), hep-ph/9711395; R. Rapp, T. Schäfer, E.V. Shuryak and M. Velkovsky, Phys. Rev. Lett. 81, 53 (1998), hep-ph/9711396.

[2] T. Schäfer, Nucl. Phys. A638, 511C (1998); M. Alford, K. Rajagopal and F. Wilczek, Nucl. Phys. A638, 515C (1998), hep-ph/9802284; K. Rajagopal, Prog. Theor. Phys. Suppl. 131, 619 (1998), hep-ph/9803341; J. Berges and K. Rajagopal, Nucl. Phys. B538, 215 (1999), hep-ph/9804233; T. Schäfer, Nucl. Phys. A642, 45 (1998), nucl-th/9806064; S. Hands and S.E. Morrison [UKQCD Collaboration], Phys. Rev. D59, 116002 (1999), hep-lat/9807033; K. Rajagopal, Nucl. Phys. A642, 26 (1998), hep-ph/9807318; N. Evans, S.D.H. Hsu and M. Schwetz, Nucl. Phys. B551, 275 (1999), hep-ph/9808444; S. Morrison [UKQCD Collaboration], Nucl. Phys. Proc. Suppl. 73, 480 (1999), hep-lat/9809040; M. Alford, Nucl. Phys. Proc. Suppl. 73, 161 (1999), hep-lat/9809166. T. Schäfer and F. Wilczek, Phys. Lett. B450, 325 (1999), hep-ph/9810509; N. Evans, S.D.H. Hsu and M. Schwetz, Phys. Lett. B449, 281 (1999), hep-ph/9810514; R.D. Pisarski and D.H. Rischke, Phys. Rev. Lett. 83, 37 (1999), nucl-th/9811104; K. Langfeld and M. Rho, hep-ph/9811227; J. Berges, D.U. Jungnickel and C. Wetterich, hep-ph/9811387; D.T. Son, Phys. Rev. D59, 094019 (1999), hep-ph/9812287; A. Chodos, H. Minakata and F. Cooper, Phys. Lett. B449, 260 (1999), hep-ph/9812305; G.W. Carter and D. Diakonov, Phys. Rev. D60, 016004 (1999), hep-ph/9812445; D. K. Hong, Phys. Lett. B 473, 118 (2000) [hep-ph/9812510]. J. Hosek, hep-ph/9812515; D. K. Hong, Nucl. Phys. B 582, 451 (2000) hhepph/9905523]. , [For a recent review see: T. Schafer and E. Shuryak, nuclth/0010049 and K. Rajagopal and F. Wilczek, hep-ph/0011333. ].

[3] M. Alford, K. Rajagopal and F. Wilczek, Nucl. Phys. B 537, 443 (1999) hep-ph/9804403.

[4] K. Rajagopal and F. Wilczek, Phys. Rev. Lett. 86, 3492 (2001) hep$\mathrm{ph} / 0012039$.

[5] D. K. Hong, M. Rho and I. Zahed, Phys. Lett. B 468, 261 (1999) hhepph/9906551. 
[6] R. Casalbuoni and R. Gatto, Phys. Lett. B 464, 111 (1999) hepph/9908227.

[7] D. T. Son and M. A. Stephanov, Phys. Rev. D 61, 074012 (2000) hepph/9910491; erratum, ibid. D 62, 059902 (2000) hep-ph/0004095.

[8] M. Rho, A. Wirzba and I. Zahed, Phys. Lett. B 473, 126 (2000) hepph/9910550.

[9] D. K. Hong, T. Lee and D. Min, Phys. Lett. B 477, 137 (2000) hepph/9912531.

[10] C. Manuel and M. H. Tytgat, Phys. Lett. B 479, 190 (2000) hepph/0001095.

[11] S. R. Beane, P. F. Bedaque and M. J. Savage, Phys. Lett. B 483, 131 (2000) hep-ph/0002209.

[12] P. F. Bedaque and T. Schaefer, hep-ph/0105150.

[13] D. K. Hong, Phys. Rev. D 62, 091501 (2000) hep-ph/0006105.

[14] C. Manuel and M. H. Tytgat, Phys. Lett. B 501, 200 (2001) hepph/0010274.

[15] S. Weinberg, Trans. New York Acad. Sci. 38, 185 (1977).

[16] D. T. Son, Phys. Rev. D 59, 094019 (1999) hep-ph/9812287.

[17] S. R. Beane, P. F. Bedaque and M. J. Savage, Nucl. Phys. A 688, 931 (2001) [nucl-th/0004013.

[18] D. B. Kaplan and A. E. Nelson, Phys. Lett. B 175, 57 (1986).

[19] A. E. Nelson and D. B. Kaplan, Phys. Lett. B 192, 193 (1987).

[20] A. Burrows and J. M. Lattimer, Astrophys. J. 307, 178 (1986). W. Keil and H. T. Janka, Astron. Astrophys. 296, 145 (1995). J. A. Pons, S. Reddy, M. Prakash, J. M. Lattimer and J. A. Miralles, Astrophys. J. 513, 780 (1999) astro-ph/9807040. 


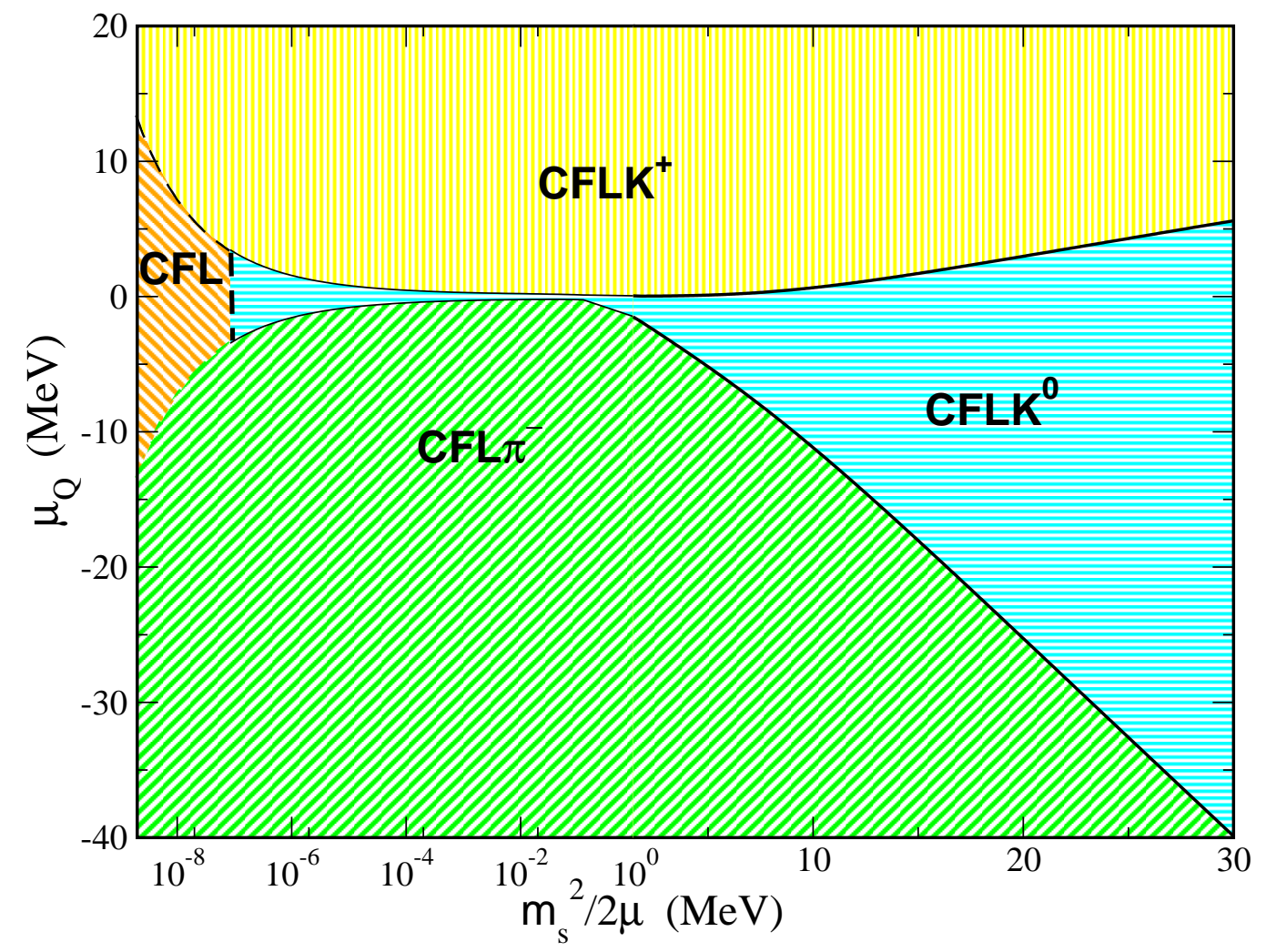

Figure 1: Meson condensed phases in the neighborhood of the symmetric CFL state are shown in the $\left(m_{s}^{2} / 2 \mu\right)-\mu_{Q}$ plane, where $m_{s}$ is the strange quark mass (set to $150 \mathrm{MeV}$ ), $\mu$ is the quark number chemical potential, and $\mu_{Q}$ is the chemical potential for positive electric charge. At five times nuclear density $\mu \sim 400 \mathrm{MeV}$ and $\left(m_{s}^{2} / 2 \mu\right) \sim 25 \mathrm{MeV}$. Solid and dashed lines indicate first- and second-order transitions respectively. 


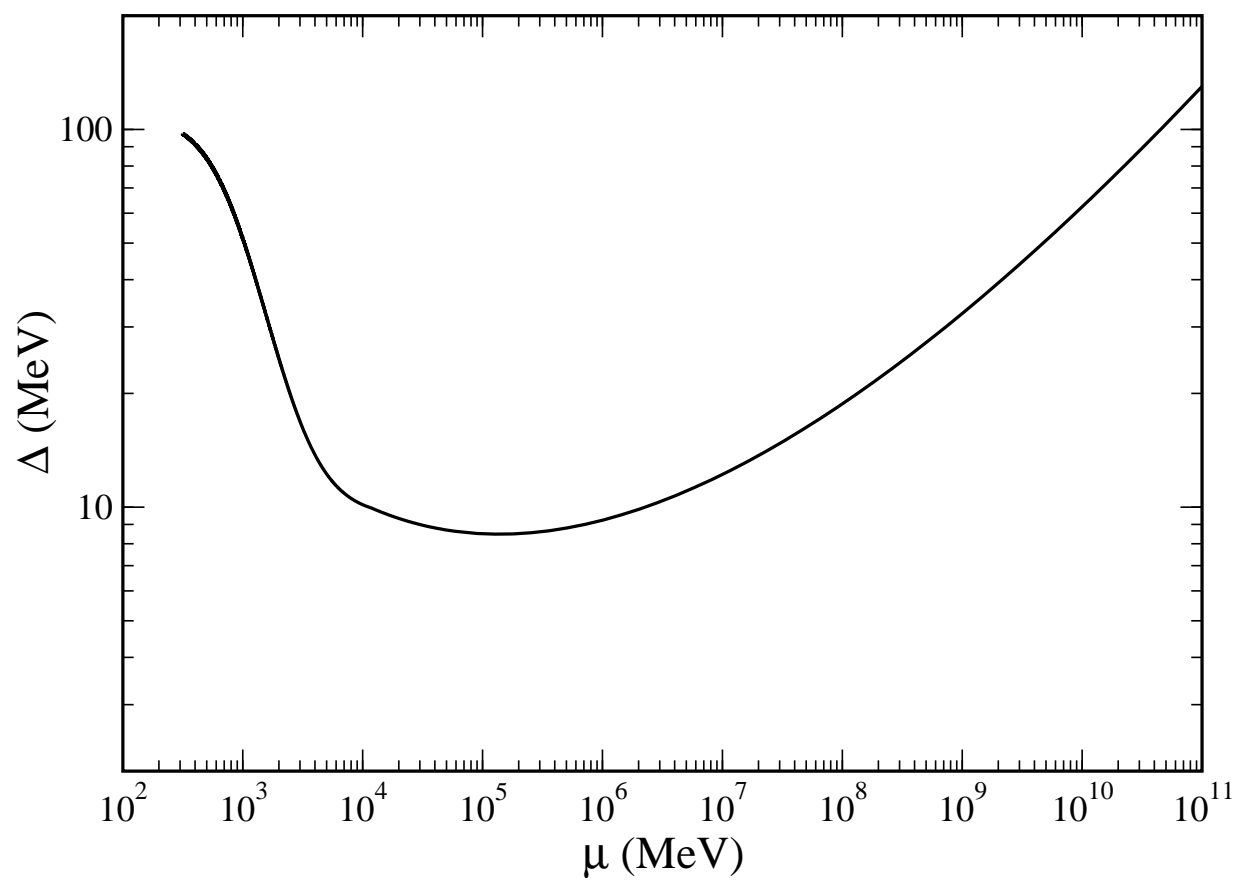

Figure 2: A model for superconducting gap $\Delta$ as a function of the quark chemical potential $\mu$. The curve agrees with perturbative calculations [16] for $\mu>10 \mathrm{GeV}$. 


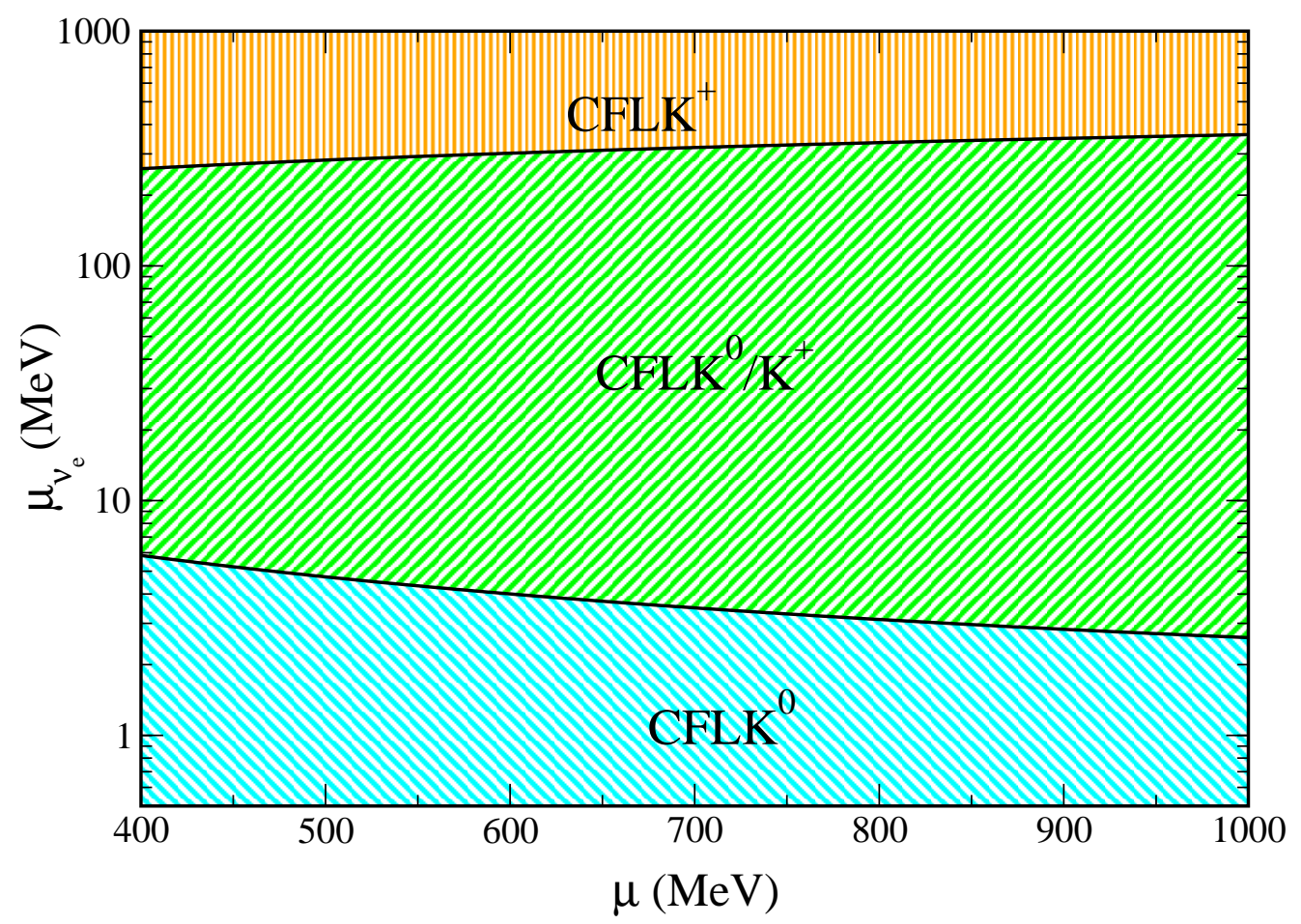

Figure 3: The phase diagram for charge neutral matter as a function of the quark number and neutrino number chemical potentials $\mu$ and $\mu_{\nu_{e}}$. The phase transitions shown are both second order. 